\title{
DESCRIPCIÓN DEL PROYECTO DE TESIS DOCTORAL: APLICACIÓN DE LA TECNOLOGIA DINSAR A LA PREVENCION DE LOS RIESGOS GEOLOGICOS NATURALES E INDUCIDOS EN CIUDADES E INFRAESTRUCTURAS PRIORITARIAS DE CENTROAMÉRICA
}

\author{
A PHD THESIS WORKING PLAN: APPLICATION OF DINSAR TECHNOLOGY TO PREVENT NATURAL AND \\ INDUCED GEOLOGICAL RISKS IN CITIES AND PRIORITY INFRASTRUCTURES IN CENTRAL AMERICA \\ Carlos García-Lanchares ${ }^{\mathrm{a}, \mathrm{b},{ }^{*},}$, Miguel Marchamalo ${ }^{\mathrm{b}}$, Candela Sancho ${ }^{\mathrm{a}}$ \\ a Detektia Earth Surface Monitoring SL. C/ Prof. Aranguren, 3, Ciudad Universitaria, 28040 Madrid, España. cglanchares@detektia.com; \\ csancho@detektia.com

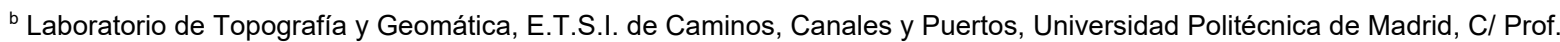 \\ Aranguren, 3, Ciudad Universitaria, 28040 Madrid, España. miguel.marchamalo@upm.es
}

\begin{abstract}
:
This communication presents the formulation and first steps of an Industrial PhD project, developed in the frame of the "Kuk ahpán" project (Spanish National R\&D Plan, 2019-2022) that aims to understand, monitor and model tectonic processes at the lithospheric scale in Central America. For this purpose, an international team from six countries (Nicaragua, Costa Rica, El Salvador, Guatemala, Norway and Spain) works integrating research in diverse geophysical techniques and engineering, with the aim of updating Seismic Risk Maps for the Region, a critical input for Safety and Construction Codes. The proposed PhD project is framed on the research and development of technologies to prevent natural and induced geological risks that affect cities and infrastructures in highly vulnerable countries, using the DInSAR (Differential Interferometry with Synthetic Aperture Radar) technology optimized by the startup Detektia Earth Surface Monitoring in collaboration with Universidad Politécnica de Madrid. Differential Synthetic Aperture Radar Interferometry is a technique based on the processing and analysis of long series of Synthetic Aperture Radar images. This technology provides records (since 1992) and up-to-date movements on any surface anywhere in the world without the need for ground instrumentation, with accuracies about $1 \mathrm{~mm} /$ year (velocity). In this context satellite radar provides valuable information on very large areas that complement the field work and in-situ instrumentation. The methodological approach starts by integrating DInSAR data with geophysical and geodesical data such as bathymetry, geomagnetism, gravimetry, seismic profiles, GNSS series... to fully map the Swan fault over Honduras and Guatemala. We use this technology to address the seismic risk over the fault and nearby areas. Then, on a second step, we will apply this seismic risk assessment (including natural and anthropogenic hazards) in prioritized cities and critical infrastructures in Central America.
\end{abstract}

Key words: DInSAR, remote sensing, geological risk, cities sustainable development, Central America

Resumen:

Este documento presenta la formulación y primeros pasos de un proyecto de Doctorado Industrial, desarrollado en el marco del proyecto Kuk ahpán que tiene como objetivo comprender, monitorear y modelar procesos tectónicos a escala litosférica en Centroamérica. Para ello, un equipo internacional de seis países (Nicaragua, Costa Rica, El Salvador, Guatemala, Noruega y España) trabaja integrando la investigación en diversas técnicas e ingenierías Geofísicas, con el objetivo de actualizar los Mapas de Riesgo Sísmico de la Región, un insumo crítico. para los códigos de seguridad y construcción. El proyecto de doctorado propuesto se enmarca en la investigación y desarrollo de tecnologías para prevenir los riesgos geológicos naturales e inducidos que afectan a ciudades e infraestructuras en países altamente vulnerables, utilizando la tecnología DInSAR (Differential Interferometry with Synthetic Aperture Radar) optimizada por la startup Detektia Earth Surface Monitoring en colaboración con la Universidad Politécnica de Madrid. La interferometría diferencial de radar de apertura sintética es una técnica basada en el procesamiento y análisis de series largas de imágenes de radar de apertura sintética. Esta tecnología proporciona registros (desde 1992) y movimientos actualizados en cualquier superficie en cualquier parte del mundo sin necesidad de instrumentación terrestre, con precisiones de alrededor de $1 \mathrm{~mm}$ / año (velocidad). En este contexto, el radar satelital proporciona información valiosa sobre áreas muy grandes que complementan el trabajo de campo y la instrumentación in situ. Primero, comenzamos integrando datos DInSAR con diversos datos geofísicos como batimetría, geomagnetismo, gravimetría, perfiles sísmicos... para mapear completamente la falla Swan sobre Honduras y Guatemala. Usamos esta tecnología para abordar el riesgo sísmico sobre la falla y áreas cercanas. En un segundo paso, aplicaremos esta evaluación de riesgo sísmico (incluyendo amenazas naturales y antropogénicas) en ciudades e infraestructuras críticas en Centroamérica.

Palabras clave: DInSAR, teledeteccion, riesgo geologico, ciudades, desarrollo sostenible, Centroamérica

*Corresponding Author: Carlos García-Lanchares, cglanchares@detektia.com 


\section{Introducción}

Los trabajos derivados de la tesis doctoral descrita en esta publicación están contextualizados dentro del proyecto Kuk ahpán y de la actividad de $1+D+i$ de la empresa Detektia. Esto es consecuencia del carácter industrial que tiene la beca doctoral otorgada por la Comunidad de Madrid en la convocatoria general del año 2020. El principal objeto de dicha convocatoria es promover el desarrollo de proyectos de Doctorado Industrial en cualquier ámbito de conocimiento y en cualquier sector empresarial.

Esta propuesta se enmarca en la investigación y desarrollo de tecnologías para combatir los riesgos geológicos naturales e inducidos que afectan a las ciudades y las infraestructuras en países de alta vulnerabilidad, mediante la tecnología DInSAR (Interferometría Diferencial con Radar de Apertura Sintética) optimizada y adaptada por la empresa tecnológica Detektia en colaboración con la Universidad Politécnica de Madrid. Esta técnica permite cuantificar la deformación de la superficie del terreno e infraestructuras a partir de imágenes adquiridas desde satélite por sensores activos en la región del espectro de las microondas (García-Sánchez et al. 2018). Detektia es una compañía de Observación de la Tierra 2.0, que utiliza datos adquiridos por satélites para detectar movimientos milimétricos en cualquier superficie e infraestructura del planeta. Asimismo, Detektia desarrolla algoritmos avanzados de Deep Learning para detectar anomalías durante toda la vida útil (desde la fase de diseño hasta la de explotación) de infraestructuras como presas, puentes, túneles, o monitorización de acuíferos y otros reservorios como petróleo y gas. Se trata de un desarrollo puntero en Europa que proporciona soluciones al problema del mantenimiento de ciudades e infraestructuras con un gran ahorro ambiental y económico.

El principal producto de Detektia, EyeRADAR, proporciona una herramienta informática de análisis en tiempo cuasireal de desplazamientos de ciudades e infraestructuras. Esta tecnología ha sido reconocida por la Incubadora de la Agencia Espacial Europea en la Comunidad de Madrid (ESA BIC), Top10 de la competición de startups de la ESA en 2020, Top20 en el reto del Global Infrastructure Hub 2021, finalista del reto BuildUP de Ferrovial (diciembre 2019) y ganadora del reto de anticipación del comportamiento del terreno de Sacyr en 2020, además de participar en los aceleradores europeos EIT Cliamte-KIC, PARSEC, STARTUP3 y ioT Trbe Space Endevour.

Decenas de miles de infraestructuras en todo el mundo (e incluso ciudades enteras) no tienen ningún sistema de monitorización contínua implementado. Detektia proporciona a diferentes sectores herramientas de monitorización de infraestructuras a un coste impensable hasta el momento. EyeRADAR permitirá aumentar la seguridad a través de la mejora de los sistemas de alerta temprana, el mantenimiento preventivo de las infraestructuras y la reducción de los costes en el control de estas sobre el terreno. EyeRADAR se puede personalizar para la monitorización de distintos objetivos estratégicos: acuíferos (Ezquerro et al. 2014; Béjar et al. 2017), túneles urbanos (Sillerico et al. 2010, 2015; García-Sánchez 2019), presas (Ruiz-Armenteros et al.
2018). Recientemente, Detektia ha desarrollado el producto City-EYE para el monitoreo de ciudades a distintas escalas (control general y control detallado de puntos críticos) en colaboración con la spinoff Insar.sk que incorpora los algoritmos del producto REMOTIO (Bakon et al. 2017; Sancho et al. 2020).

Actualmente, el control de infraestructuras se realiza con métodos geotécnicos y geodésicos tradicionales (regletas, inclinómetros, acelerómetros, redes GNSS, etc..). Esta propuesta desarrollará en Centroamérica sistemas de monitorización de altísimo detalle a un coste económico muy competitivo para aquellas instalaciones e infraestructuras que no poseen un sistema de control. Asimismo, permitirá mejorar la monitorización en aquellas en las que ya hay instalados métodos tradicionales.

Las ventajas diferenciales que ofrecen los sistemas EyeRADAR con respecto a los métodos tradicionales son:

- Precisión milimétrica, actual e histórica: series temporales de los movimientos del terreno e infraestructuras con precisión milimétrica. Esta tecnología permite analizar movimientos actuales y compararlos con deformaciones pasadas 0 históricas. En muchas regiones geográficas hay datos desde 1992 que permiten crear y analizar series temporales de los movimientos a largo plazo de regiones concretas y de sus áreas próximas, aportando información crítica que hace posible preveer el comportamiento antes del inicio de la ejecución de obra.

- Actualizaciones constantes: Las mediciones de los movimientos se actualizan tan a menudo como los satélites adquieren una imagen (desde pocos días a semanas según las necesidades).

- Sin necesidad de instrumentación en tierra: La tecnología emergente DInSAR ha demostrado su capacidad para detectar y medir pequeñas variaciones milimétricas en cualquier superficie sin necesidad de instrumentación en tierra.

- $\quad$ Alta densidad de puntos de control: Dependiendo de la superficie de estudio y de las imágenes de satélite disponibles, obtenemos un aumento exponencial de la densidad de puntos de seguimiento comparado con las campañas de instrumentación tradicional.

La presente propuesta responde al reto de desarrollar herramientas que permitan prevenir y reducir los riesgos que amenazan el desarrollo en países de Centroamérica y Caribe. Asimismo, la tecnología desarrollada será promocionada a nivel internacional para su escalado y aplicación masiva en entornos con problemática similar.

\section{Objetivos}

Los objetivos planteados para la presente tesis son los siguientes:

1) Validar el empleo de la tecnología DInSAR para la prevención de riesgos geológicos en infraestructuras prioritarias de Centroamérica.

La tecnología DInSAR implica varios avances. No obstante, para garantizar que la simbiosis entre la sismología y dicha tecnología es valida, este 
ejercicio plantea el análisis en casos a escala regional, considerando datos topográficos y geodésicos.

Dicho análisis consistirá en estimar la incertidumbre de las series de velocidades DInSAR y de la velocidad calculada por esta técnica.

2) Integrar la técnica DInSAR en un sistema de monitoreo regional que permita reducir la incertidumbre en la prevención de riesgos ligados a la tectónica de placas.

Este objetivo lleva implícito un proceso de replicación a una escala menor que consiste en pasar de la información regional generada y validada en el objetivo anterior. Basada en la priorización de las zonas de riesgo y de las intensidades máximas que se predigan. Se puede priorizar la vigilancia en las infraestructuras más vulnerables, esto se traduce en la mejora de la prevención de siesgos geológicos naturales.

3) Diseñar un sistema de control integrado con DInSAR para una infraestructura urbana o interurbana crítica en Centro América.

El último objetivo trataría la integración del sistema de monitorio con la plataforma EyeRadar y su promoción en los países de la zona de estudio. En esta fase, se espera integrar los resultados de otra línea de investigación, sobre la puesta a punto de sistemas de alerta temprana empleando inteligencia artificial. El sistema integrado será evaluado en términos técnicos y económicos, incluyendo el análisis de la sostenibilidad del sistema de monitorización.

\section{3. Área de estudio}

El área de estudio seleccionada es la Región Centroamericana. Es un área que, por su tectónica y la cantidad de estructuras sísmicas (ver Figura 1) además del gran vacío de datos que existe, la convierten en una zona de especial interés para el estudio. Una región caracterizada por la interacción de las placas de Cocos, Caribe, Nazca, la microplaca de Panamá y las placas de Norte América y Sud América (Benito et al. 2018).

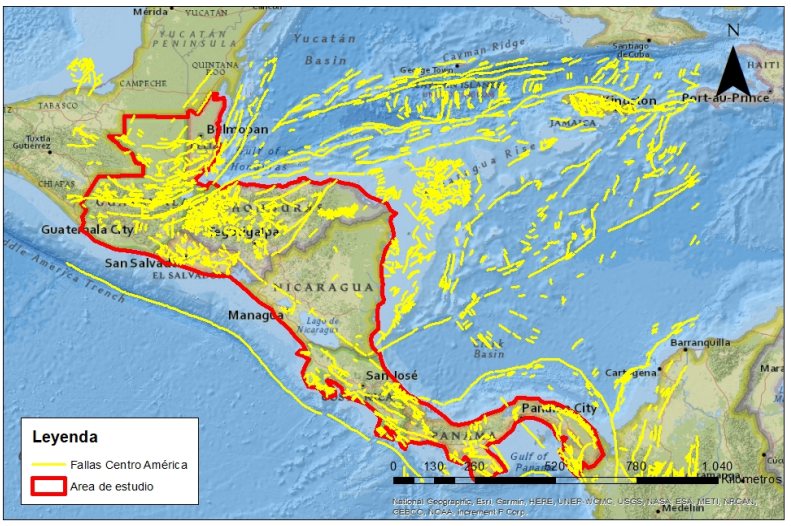

Figura 1: Área de estudio del Proyecto.Fuente: Esri (2019).

En el ámbito de las investigaciones de las deformaciones de la corteza con técnicas GNSS, existe una carencia de datos en la zona de Centroamérica y en especial en Honduras. La bibliografiá existente indica que hay velocidades de unos $15 \mathrm{~mm} / \mathrm{año}$ de Centro América moviéndose en la dirección oeste noroeste con relación a la Placa Caribe. Este dato resulta del estudio realizado con datos geodésicos, estructurales y paleomagnéticos, combinados con datos GPS de Guatemala, El Salvador y sur de Honduras (Benito and Torres 2010) de los antearcos de Nicaragua y El Salvador. Aparte de este trabajo, no se han encontrado otros estudios en la zona del Golfo de Fonseca ni en la zona del bloquede Chortís en el Atlántico. El proyecto Kuk ahpán permitirá cubrir este vacío de conocimiento complementando y validando los resultados de las mediciones publicadas en 2011 (Benito et al. 2018).

\section{Metodología}

La metodología descrita en este documento se divide en dos fases: de modelización sísmica y DInSAR. (Ver Figura 2).

\subsection{Fase modelización sísmica}

El primero de los pasos corresponde a la actividad de investigación sismológica que parte a su vez de las campañas de campo (Subproyecto 1 del proyecto Kuk Ahpán); dicha fase consiste en la adquisición de datos mediante diferentes campañas batimetría, campañas GPS, altimetria, etc.. El subproyecto 2 dará inició a la producción de los mapas de riesgo de las zonas de estudio: las grandes ciudades de las ciudades centro americanas.

La modelización de los escenarios sísmicos toma como base el empleo combinado de herramientas SIG, Hojas de cálculo e interpretación experta de los resultados. (Ver Figura 2A).

Modelos de aceleración. Los modelos empleados en este apartado se obtienen de una revisión bibliográfica (Abrahamson et al. 2013; Campbell and Bozorgnia 2014; Boore et al. 2014). Los investigadores del proyecto especializados en cada área de estudio aportan su conocimiento a lo largo del proyecto. Por lo tanto, los modelos empleados pueden actualizarse con el fin de conseguir resultados más veraces. Los modelos de aceleración permiten, conociendo la geometría de las estructuras sísmicas (fallas) y procesando los datos espaciales en un entorno SIG, obtener una capa raster que muestre la PGA o Peak groun Acceleration para un sismo de Magnitud máxima (según casos empíricos). La capa resultando muestra la aceleración sobre roca.

A continuación, basando la decisión en criterio experto, se ejecuta una ponderación sobre los distintos modelos para combinarlos y obtener un único resultado por falla, este resultado también se apoyará en análisis Radar que consistirá en interferometrías realizadas en distintos episodios sísmicos

Mapa geológico de la zona. Empleando la información de NEHRP (National earthquake Hazards reuction program) y la cartografía de tipos de suelo de cada subárea de estudio. Se combinan las PGA con los factores de amplificación para conocer la aceleración real sobre el suelo. (FEMA 2015; Schmidt et al. 2005). 


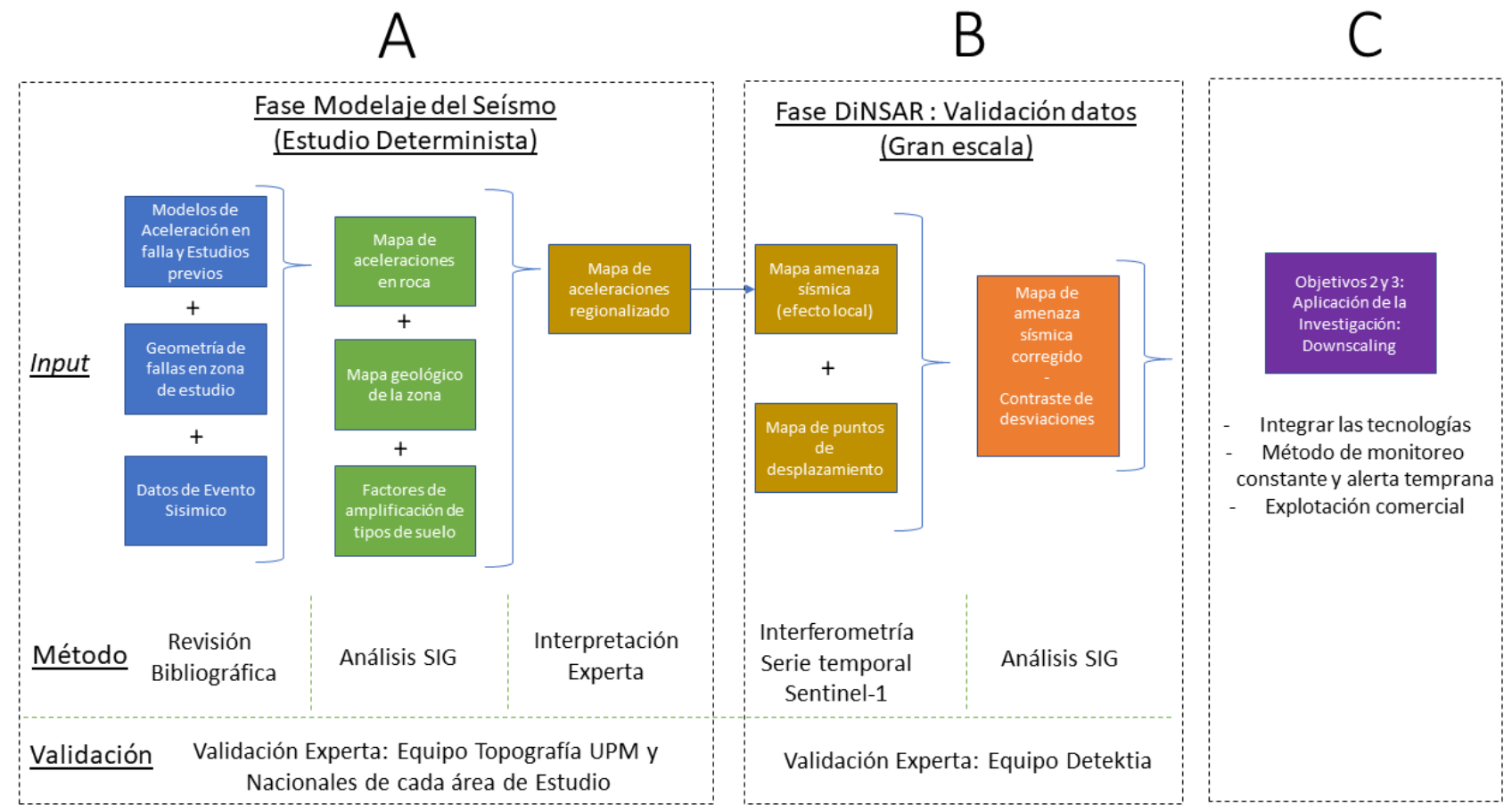

Figura 2: Esquema de la metodología.

\subsection{Fase DInSAR}

En esta segunda fase se emplea la tecnología DInSAR para validar los datos resultantes de la fase anterior (ver Figura 2B).

Se proponen las siguientes actividades:

1) Integración de los datos de auscultación disponibles, especialmente campañas GPS/GNSS, en una base de datos del proyecto.

2) Armonización y depurado de las series de datos GPS/GNSS.

3) Obtención del catálogo de imágenes SAR correspondiente a las series de datos disponibles empleando sensores actuales (i.e. embarcados en Sentinel 1 A/B-ver Tabla 1) o históricos (ERS 1 / 2, ENVISAT).

4) Procesado DInSAR de las imágenes SAR empleando la tecnología desarrollada por Detektia.

5) Validación de velocidades de deformacion observadas por técnica DInSAR con la base de datos de ascultación.

6) Estimación de la incertidumbre de las series de velocidades.

Las series temporales de la cartografía utilizada para detectar los movimientos, de naturaleza radar y óptica, se procesarán utilizando la tecnología de Detektia (ordenadores y código). La misma tendrá un proceso de validación con datos de campo cuya información se obtendrá de las redes GNSS/GPS disponibles y las estaciones nuevas que instalará el proyecto Kuk ahpán (red de 10 estaciones GPS en el borde hondureño de la falla) que monitorizan de forma continua las posiciones durante el tiempo de ejercicio del proyecto.
Tabla 1: Template Imagenes de Sentinel-1A disponibles desde Diciembre 2014 hasta 13/05/2021. Fuente: ESA (2021).

\begin{tabular}{c|c|c} 
País & $\begin{array}{c}\text { Geometria } \\
\text { ascendiente }\end{array}$ & $\begin{array}{c}\text { Geometria } \\
\text { descendente }\end{array}$ \\
\hline Costa Rica & 6.066 & 6.808 \\
Guatemala & 15.066 & 15.128 \\
Nicaragua & 10.314 & 10.221 \\
El Salvador & 5.819 & 5.927 \\
Honduras & 8.835 & 9.076 \\
Panamá & 9.437 & 8.399
\end{tabular}

\subsection{Metodología de los años sucesivos}

Las actividades propuestas variarán dependiendo de los resultados y necesidades que se observen en el primer año de trabajo.

En un contexto ideal, se procedería con la integración de los datos observados en la tecnología EyeRADAR de Detektia. Algunas tareas proyectadas son:

1) Validar EyeRADAR con datos disponibles.

2) Integrar EyeRADAR en el sistema de monitorización del proyecto Kuk ahpán.

3) Estimar la mejora de la prevención de riesgos geológicos naturales $\mathrm{e}$ inducidos mediante la incorporación de la técnica DInSAR a los sistemas propuestos por el proyecto Kuk ahpán.

4) Integrar el análisis por Inteligencia Artificial (IA) de los datos proporcionados por EyeRADAR para identificar patrones de riesgo en las series de desplazamiento.

5) Seleccionar una ciudad prioritaria en coordinación con las instituciones participantes en el proyecto 
6) Colaborar en el diseño de la base de datos de monitorización

7) Desarrollar el producto City-EYE para el caso de estudio elegido

8) Participar en la difusión de los resultados del proyecto Kuk ahpán.

9) Colaborar en la acción comercial de Detektia en Centroamérica.

El riesgo sísmico se analizará posteriormente en zonas de riesgo ejemplo, teniendo como objetivo la exposición sistemática de las pérdidas económicas, de infraestructura y el número de vidas afectadas.

\section{Resultados}

Los resultados esperados se están predispuestos en tres bloques.

\subsection{Validación del mapa de amenaza sísmica}

El primer producto que se conseguiría empleando el método descrito, sería un mapa de peligrosidad contrastado. Los modelos sísmicos empleados proporcionan un abanico de resultados, que si pudieran apoyarse en los datos InSAR para hacer confirmar su validez propiciaría una estrategia civil certera. En la Figura 3 se pueden ver los desplazamientos en la dirección de mira del satélite (geometría ascendente) de San José para el periodo 2015 y 2021. El resultado final será la relación entre este mapa de velocidades y el mapa de aceleraciones.

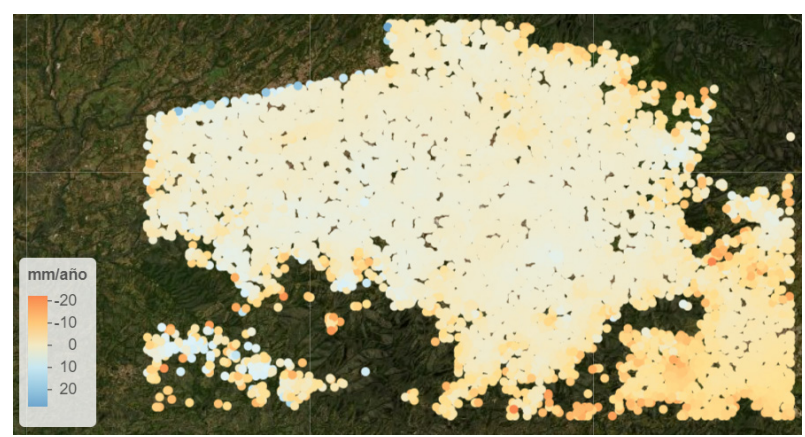

Figura 3: Ejemplo de desplazamiento en San José (Costa Rica) Julio, 2015-Enero, 2021. Serie temporal de 96 imágenes (60.787 puntos). Fuente: Detektia (2021) and STYRON (2019)

\subsection{Downscalling: análisis posteriores}

Las dos preguntas que queremos responder antes de hacer el ya seleccionado downscalling son: ¿Dónde puede haber un detonante? y ¿Qué intensidad tendrá éste?; es decir, que zonas son susceptibles de sufrir un movimiento sísmico de una intensidad significativa. Una vez conocidas dichas áreas sabremos dónde hay que observar a una escala menor, dónde están los lugares de interés.

Los casos de aplicación directa, una vez conocidas las infraestructuras pueden ser de diversa naturaleza: carreteras que puedan ser afectadas por derrumbamientos, grandes acuíferos con variaciones estacionales que puedan comprometer la estabilidad de estructuras superficiales, obras subterráneas o edificios.
En el caso de la Figura 4 se puede observar una carretera en Colombia en una zona montañosa en la que se detectan movimientos de hasta 13 milímetros/año. La monitarización en tiempo real puede ser posible combinando los resultados obtenidos por DInSAR con Inteligencia artificial, considerando variables como la precipitación, la temperatura, topografía, etc..

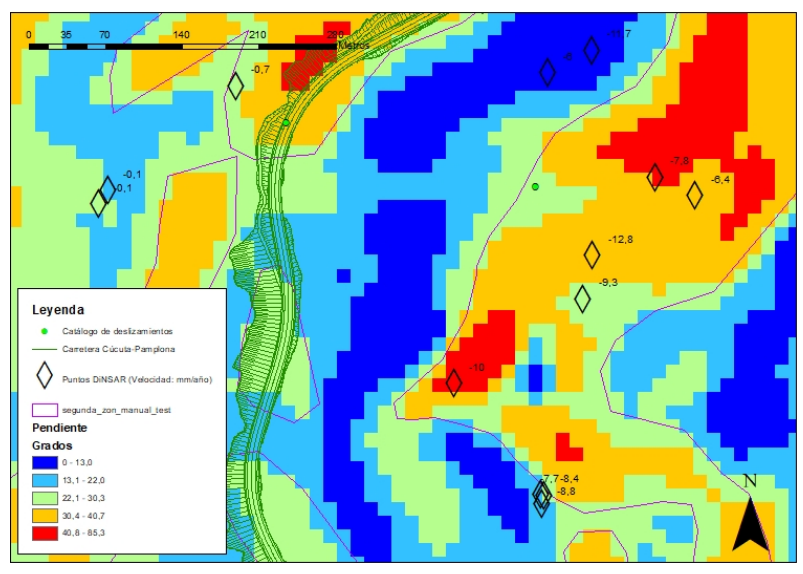

Figura 4: Análisis multivariable preliminar de deslizamientos en entorno de infraestructura vulnerable.

\subsection{Ventajas de la aplicación de la técnica DInSAR a la prevención de los riesgos naturales inducidos}

Las ventajas que se preveen al aplicar la tecnología DInSAR en el ámbito de estudio descrito son las siguientes:

1) Social. Existe un impacto de la tecnología también desde la perspectiva social. El riesgo de desastre debido a causas naturales o tecnológicas puede minimizarse gracias al desarrollo de soluciones tecnológicas de vigilancia. En todo caso, lo que conseguimos con el estudio de cambios a nivel milimétrico es reducir la vulnerabilidad como determinante del colapso de infraestructuras.

2) Económica. Como se ha descrito, la monitorización de infraestructuras empleando tecnología radar satelital supone ahorros sustanciales, tanto gastos de personal que tienen que desplazarse hacia las infraestructuras para realizar las mediciones, como los ahorros en costes inherentes del mantenimiento preventivo. Asimismo, es importante considerar el ahorro derivado de evitar potenciales colapsos o fallos irreversibles en infraestructuras.

Inmediato: El ahorro en los proyectos civiles se contempla en dos aspectos: campañas de campo en la obtención de datos y acceso a los lugares de la obra en situaciones de difícil acceso.

Plazo no inmediato: En el caso de que ocurra un evento sísmico, y dependiendo de la intensidad de este, las pérdidas económicas son variables. Estas son las derivadas de volver a habilitar la infraestructura afectada. El ahorro derivado de la monitorización a medio/largo plazo se produciría teniendo en cuenta los datos de peligrosidad sísmica en la planificación urbanística, tanto en la selección de los emplazamientos como en el tipo de construcciones. 
3) Medioambiental. Un control preventivo y eficaz de infraestructuras puede suponer una reducción sustancial de emisiones de gases de efecto invernadero, al evitar colapsos y daños irreversibles disminuimos la necesidad de obra nueva y en consecuencia se reducen las emisiones de GEI.

\section{Conclusiones}

La interferometría diferencial de radar de apertura sintetica (DInSAR) es una técnica de teledetección que garantiza la calidad de su empleo en la ingeniería civil (García-Sánchez 2019; Cigna and Tapete 2021).
La presente propuesta responde al reto de desarrollar herramientas que permitan prevenir y reducir los riesgos que amenazan el desarrollo en países de Centroamérica.

Asimismo, la tecnología desarrollada será promocionada a nivel internacional para su escalado y aplicación masiva en entornos con problemática similar.

\section{Agradecimientos}

Esta línea de investigación ha sido desarrollada con el apoyo de los equipos de Detektia Earth Surface Monitoring SL, la E.T.S.I. de Caminos, Canales y Puertos de la Universidad Politécnica de Madrid y el equipo de trabajo de Kuk ahpán.

\section{References}

ABRAHAMSON, N.A., SILVA, W.J., and KAMAI, R., 2013. Update of the AS08 Ground-Motion Prediction Equation Based on the NGA-West2 Data Set, Pacific Earthquake Engineering Research Center University of California, Berkeley, PEER 2013/04

BAKON, M., I. OLIVEIRA, D. PERISSIN, J. J. SOUSA, and PAPCO, J., 2017. A Data Mining Approach for Multivariate Outlier Detection in Postprocessing of Multitemporal InSAR Results. IEEE Journal of Selected Topics in Applied Earth Observations and Remote Sensing, 10(6), pp. 2791-2798. DOI: 10.1109/JSTARS.2017.2686646

BEJAR-PIZARRO, M., EZQUERRO, P., HERRERA, G., TOMÁS, R., GUARDIOLA, ALBERT, C., RUIZ HERNÁNEZ, J.M., FERNÁNDEZ-MERODO, J.A., MARCHAMALO, M., and MARTINEZ, R., 2017. Mapping groundwater level and aquifer storage variations from InSAR measurements in the Madrid aquifer, Central Spain. Journal of Hydrology. DOI: 10.1016/j.jhydrol.2017.02.011

BENITO, B., and TORRES, Y., 2010. Amenaza sísmica en América central. Entimema, Madrid.

BENITO, B., CORDOBA, and BARBA, D., 2018. Kuk ahpán: Estudio de regional integrado de la estructura y evolución 4D de la litofera en América Central. Implicaciones en el calculo de la amenaza y riesgo sismico (Kuk Ahpán). Memoria Científico Técnica de proyectos coordinados. Agencia Estatal de Investigaciobn- Convocatoria 2018 Proyectos de I+D de Generacion de Conocimiento y Proyectos de I+D+i Restos Investigación. Ministerio de Ciencia, Innovación y Universidades.

BOORE, D.M., STEWART, J.P., EEMEL, SEYHAN, M., and ATKINSON, G.M., 2014. NGA-West 2 Equations for Predicting PGA, PGV, and 5\% Damped PSA for Shallow Crustal Earthquakes,

CAMPBELL, K.W., and BOZORGNIA, Y., 2014. Campbell-bozorgnia NGA-West2 horizontal ground motion model for active tectonic domains, COrelogic, Inc., University of California, Berkeley

CIGNA, F., TAPETE, D., 2021. Sateliite InSAR Survey of structurally controlled land subsidence due to groundwater exploitation in te Aguascalientes Valley, Mexico. ELSEVIER, Remote Sensing of Environment, 254, 1 March 2021, 112254

DETEKTIA. 2021. Tecnología InSAR. Detectamos desde el espacio desplazamientos milimétricos de la superficie terrestre. Available: https://detektia.com/ [7/23, 2021].

ESA. 2021. European Space Agency - Spain. Available: https://www.esa.int/Space_in_Member_States/Spain [7/23, 2021].

ESRI. 2019. Mapa de estilo National Geographic. Última actualización: 30 Sept. 2020

EZQUERRO, P., HERRERA, G., MARCHAMALO, M., TOMÁS, R., BEJARPIZARRO, M., MARTíNEZ, and MARÍN, R., 2014. A quasielastic aquifer deformational behavior: Madrid aquifer case study. Journal of Hydrology, 519(2014), pp. 1192-1204.

FEMA. 2015. NEHRP Recommended Seismic Provisions for New Buildings and Other Structures, Volume I: Part 1 Provisions, Part 2 Commentary FEMA P-1050-1/2015 Edition, National Earthquake Hazard Reduction Program

GARCIA-SANCHEZ, A.J., BAKON, M., MARTINEZ, R., and MARCHAMALO, M., 2018. Evolution of urban monitoring with radar interferometry in Madrid City: performance of ERS-1/ERS-2, ENVISAT, COSMO-SkyMed, and Sentinel-1 products. International Journal of Remote Sensing, 39(9), pp. 2969-2990.

GARCIA-SANCHEZ, A.J., 2019. Application of differential Synthetic Aperture Radar Interferometry (DiNSAR) to the monitoring of ground and structure movements. Tesis Doctoral, ETSI Caminos, Canales y Puertos. Laboratorio de topografia y Geomática. Doctorado en Ingenieriá de Estructuras, Cimentaciones y Materiales, Madrid

RUIZ-ARMENTEROS, A.M., LAZECKY, I., HLAVÁCIVÁ, B., DELGADO, J.M., SOUSA LAMAS-FERNANDEZ, L., MARCHAMALO, M., CAROCUENCA, M., PAPCO, J., and PERISSIN D., 2018. Deformation monitoring of dam 
infrastructures via spaceborne MT-InSAR. The case of La Viñuela (Málaga, southern Spain). Procedia Computer Science, 138(2018), pp. 346-353

SANCHO, C., BAKON, M., GARCIA, A.J., CZIKHARDT, R., FERNANDEZLANDA, A., PAPCO, J., MARTINEZ R. BARLAK, J., MARCHAMALO, M., ROVNAK, M., and ADAMISIN, P., 2020. City-EYE a platform for the surveillance and management of built-up area and infrastructures. Procedia Computer Science, (aceptado, 2020) ISSN 1877-0509

SILLERICO, E., MARCHAMALO, M., REJAS, J.G., and MARTINEZ, R., 2010. La técnica DInSAR: bases y aplicación a la medición de subsidencias del terreno en la construcción. Informes de la Construcción, 62(519), pp. 47-53. ISSN 00200883

SILLERICO, E., P. EZQUERRO, M. MARCHAMALO, M., HERRERA, G., DURO, J., and MARTíNEZ, R., 2015. Monitoring ground subsidence in urban environments: M-30 tunnels under Madrid City (Spain). Ingeniería e Investigación, 35(2) (Agosto 2015). ISSN: 0120-5609.

SCHMIDT, V., MOYA, A., CLIMENT, ROJAS, A., WILFREDO, R., and BOSCHINI, I., 2005. Microzonificación sísmica de San José, Costa Rica. Editorial de la universidad de Costa Rica, ISBN: 9977-67-963-0

STYRON, R., 2019. GEMScienceTools/gem-global-active-faults: First release of 2019 (Version 2019.0). Centre for Observation and Modelling of Earthquakes, Volcanoes and Tectonics (COMET). Global active faults data. Zenodo. http://doi.org/10.5281/zenodo.3376300. Modification: lines are simplified). Volcanoes database, Tiles @ Esri - Source: Esri, i-cubed, USDA, USGS, AEX, GeoEye, Getmapping, Aerogrid, IGN, IGP, UPR-EGP, and the GIS User Community 